\title{
High specificity of a novel Zika virus ELISA in European patients after exposure to different flaviviruses
}

D Huzly ${ }^{1}$, I Hanselmann ${ }^{1}$, J Schmidt-Chanasit ${ }^{23}$, M Panning ${ }^{1}$

1. Institute for Virology, Medical Center - University of Freiburg, Freiburg, Germany

2. Bernhard Nocht Institute for Tropical Medicine, WHO Collaborating Centre for Arbovirus and Haemorrhagic Fever Reference and Research, National Reference Centre for Tropical Infectious Diseases, Hamburg, Germany

3. German Centre for Infection Research (DZIF), partner site Hamburg-Luebeck-Borstel, Hamburg, Germany

Correspondence: Marcus Panning (marcus.panning@uniklinik-freiburg.de)

Citation style for this article:

Huzly D, Hanselmann I, Schmidt-Chanasit J, Panning M. High specificity of a novel Zika virus ELISA in European patients after exposure to different flaviviruses. Euro Surveill. 2016;21(16): pii=30203. DOI: http://dx.doi.org/10.2807/1560-7917.ES.2016.21.16.30203

Article submitted on 30 March 2016 / accepted on 21 April 2016 / published on 21 April 2016

The current Zika virus (ZIKV) epidemic in the Americas caused an increase in diagnostic requests in European countries. Here we demonstrate high specificity of the Euroimmun anti-ZIKV IgG and IgM ELISA tests using putative cross-reacting sera of European patients with antibodies against tick-borne encephalitis virus, dengue virus, yellow fever virus and hepatitis $C$ virus. This test may aid in counselling European travellers returning from regions where ZIKV is endemic.

Current interim guidelines in Europe for symptomatic patients and pregnant women returning from regions endemic for Zika virus (ZIKV) recommend serological testing from day 5 after onset of disease [1]. However, serological diagnosis remains challenging because of extensive cross-reactivity between antibodies against flaviviruses [2]. In Europe, tick-borne encephalitis virus (TBEV) is the most relevant flavivirus and might cause diagnostic problems in sera from European travellers returning from ZIKV endemic regions. Recently, an ELISA based on ZIKV NS1-antigen has been developed and shown to diagnose ZIKV infections [3]. Here, we evaluated the specificity of this novel ZIKV ELISA using sera from European patients with laboratory-confirmed and putative cross-reacting antibodies against different flaviviruses and other acute viral infections.

\section{Human serum samples}

Samples with a high potential of causing cross-reactions in serological flavivirus assays were chosen: acute TBEV infection, acute dengue virus infection, recently boostered tick-borne encephalitis (TBE) vaccination with high levels of TBEV IgG, recent yellow fever vaccination and viraemic hepatitis $C$ virus (HCV) infection. TBEV, dengue and HCV sera contained laboratory-confirmed high levels of IgG antibodies against these viruses. All 26 dengue virus antibody-positive sera were from German travellers. Of these, 16 acute dengue sera were positive for anti-dengue virus IgM and for dengue virus $\mathrm{NS}_{1}$ antigen and were positive in dengue virus RT-PCR. Follow-up sera were available from 10 patients after laboratory-confirmed acute dengue infection and were anti-dengue virus IgG-positive only.

For evaluation of the ZIKV IgM ELISA, we used in addition sera from patients with polyclonal IgM stimulation (acute Eppstein-Barr virus (EBV) infection $(n=22)$, acute Mycoplasma pneumoniae infection $(n=8)$, primary cytomegalovirus (CMV) infection in pregnancy $(n=9)$, and primary human immunodeficiency virus (HIV) infection $(n=13))$. All sera were submitted to the Institute of Virology, Freiburg, for routine diagnostics and were stored at $-20^{\circ} \mathrm{C}$ in an anonymised biobank before testing.

To confirm the capability of the ZIKV ELISA to detect ZIKV antibodies, we analysed 10 patient samples from Brazil with acute or recent ZIKV infection. For laboratory confirmation of ZIKV infection in these patients we used an indirect immunofluorescent assay (IIF) as described [4]. IIF titres for anti-ZIKV IgM ranged from $1: 1,280$ to $1:>20,480$, and for anti-ZIKV IgG from 1:320 to $1:>20,480$. All 10 Brazilian sera had previously tested negative at the Bernhard Nocht Institute for Tropical Medicine for IgM and IgG against dengue virus, and negative for dengue virus NS1 antigen. In addition, two serum samples from a German tourist returning from Brazil with ZIKV infection were available to us. The first sample had been taken on day 3 after symptom onset in 2015, a second sample one year later. The first serum sample tested ZIKV RT-PCR-negative, but a saliva sample from the same day (three days after symptom onset) tested RT-PCR-positive, confirming the diagnosis of acute ZIKV infection.

\section{Laboratory investigation}

We used the Euroimmun ZIKV ELISA (Euroimmun, Lübeck, Germany) according to the manufacturer's recommendation. In brief, sera were diluted 1:101 in 

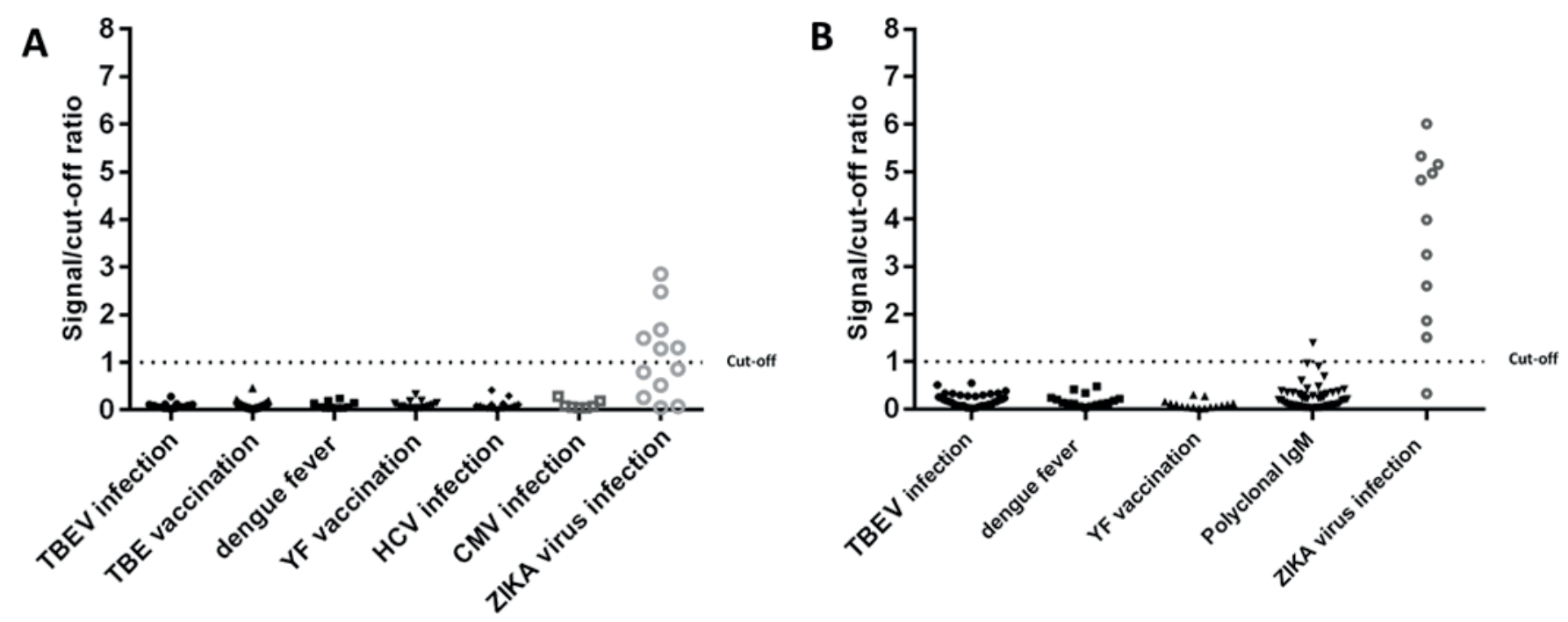

CMV: cytomegalovirus; ELISA: enzyme-linked immunosorbent assay; HCV: hepatitis C virus; TBE: tick-borne encephalitis; TBEV: tick-borne encephalitis virus; YF: yellow fever; ZIKV: Zika virus.

\section{TABLE 1}

Serological test results for different cohorts using the ZIKV IgG ELISA

\begin{tabular}{|c|c|c|c|c|c|}
\hline \multirow{2}{*}{ Cohort } & \multirow{2}{*}{ Number of samples } & \multirow{2}{*}{ Origin of infection } & \multicolumn{3}{|c|}{ Result ZIKV IgG ELISA } \\
\hline & & & Negative & Borderline & Positive \\
\hline TBEV infection ${ }^{a}$ & 21 & Germany & 21 & 0 & 0 \\
\hline TBE vaccination ${ }^{\mathrm{a}}$ & 52 & Germany & 52 & 0 & 0 \\
\hline Dengue virus infection ${ }^{b}$ & 10 & Endemic regions & 10 & 0 & 0 \\
\hline Yellow fever vaccination ${ }^{c}$ & 15 & Germany & 15 & o & 0 \\
\hline HCV infection ${ }^{d}$ & 16 & Germany & 16 & 0 & 0 \\
\hline Acute ZIKV infection ${ }^{\mathrm{e}}$ & 11 & Brazil & 5 & 1 & 5 \\
\hline Past ZIKV infection ${ }^{\mathrm{e}}$ & 1 & Brazil & 0 & 0 & 1 \\
\hline
\end{tabular}

ELISA: enzyme-linked immunosorbent assay; HCV: hepatitis C virus; IgG; immunoglobulin G; IgM: immunoglobulin M; TBE: tick-borne encephalitis; TBEV: tick-borne encephalitis virus; ZIKV: Zika virus.

a TBEV IgM and IgG detection was performed with Serion classic ELISA TBE IgM and IgG quant assay (Virion/Serion, Würzburg, Germany).

${ }^{b}$ Confirmed with SD dengue NS1 + Ab Combo (MT Promedt Consulting, St. Ingbert, Germany).

c Documented yellow fever vaccination.

${ }^{d}$ Detection of HCV antibodies was done using the Architect Anti-HCV assay (Abbott, Wiesbaden, Germany).

e Detection of ZIKV antibodies was done using IIF as described in the text.

sample buffer and incubated at $37^{\circ} \mathrm{C}$ for 60 min in a microplate well. Before IgM detection, sera were preincubated with sample buffer containing rheumatoid factor absorbent as recommended. Further steps were done as described elsewhere, and the optical density (OD) was measured in a BEP III system (Siemens Healthcare, Munich, Germany). A signal-to-cut-off ratio was calculated, and values $<0,8$ were regarded as negative, $\geq 0,8$ to $<1,1$ as borderline, and $\geq 1,1$ as positive.

\section{ZIKV IgG ELISA}

The ZIKV IgG ELISA was positive or borderline in six of 10 samples from Brazilian patients with clinical and laboratory-confirmed acute ZIKV infection (Table 1). The first sample of a German tourist tested ZIKV IgGnegative, but was ZIKV IgG-positive one year after acute ZIKV infection (past ZIKV infection, Table 1). No IgG ELISA reactivity above the threshold for positivity was seen in any of the potentially cross-reacting samples (Figure). 
Serological test results for different cohorts using the ZIKV IgM ELISA

\begin{tabular}{|l|c|c|c|c|c|}
\hline & & & \multicolumn{3}{|c|}{ Result ZIKV IgM ELISA } \\
\hline Cohort & Number of samples & Origin of infection & Negative & Borderline & Positive \\
\hline TBEV infection & 38 & Germany & 38 & 0 & 0 \\
\hline Dengue virus infection $^{\mathrm{b}}$ & 16 & Endemic regions & 16 & 0 & 0 \\
\hline Yellow fever vaccinationc & 15 & Germany & 15 & 0 & 0 \\
\hline Polyclonal IgM & 52 & Germany & 49 & 2 & 1 \\
\hline ZIKV infection & 11 & Brazil & $1^{\mathrm{d}}$ & 0 & 10 \\
\hline
\end{tabular}

ELISA: enzyme-linked immunosorbent assay; IgG; immunoglobulin G; IgM: immunoglobulin M; TBEV: tick-borne encephalitis virus; ZIKV: Zika virus.

a TBEV IgM and IgG detections were performed with Serion classic ELISA TBE IgM and IgG quant assay (Virion/Serion, Würzburg, Germany).

b SD dengue NS1 + Ab Combo (MT Promedt Consulting, St. Ingbert, Germany), RT-PCR was done using the RealStar dengue RT-PCR kit (Altona Diagnostics, Hamburg, Germany).

c Documented yellow fever vaccination.

${ }^{\mathrm{d}}$ German tourist day 3 after symptom onset.

Overall, specificity of the ZIKV IgG ELISA was $100 \%$ (95\% confidence interval: 95.9-100.0).

\section{ZIKV IgM ELISA}

All 10 sera from the Brazilian patients tested positive using the ZIKV IgM ELISA (Table 2). One sample from a German patient with a polyclonal IgM (reactivity in TBE virus IgM and EBV IgM assay) was positive in the ZIKV IgM ELISA. Two samples from patients with acute EBV infection showed borderline results in the ZIKV IgM ELISA. None of the samples from patients with acute TBE virus infection, dengue fever, or recent yellow fever vaccination showed reactivity above the threshold for positivity, demonstrating the high specificity of the Euroimmun ZIKV IgM ELISA (Figure).

\section{Discussion}

There is now evidence of a causal relationship between ZIKV infection during pregnancy and severe birth defects $[5,6]$. In Europe, laboratory diagnosis should be performed in pregnant women returning from ZIKV endemic regions [7]. Follow-up ultrasound examinations and counselling are recommended for those with markers of recent ZIKV infection. In light of the possible severe consequences for pregnant women and their fetus, it is imperative that serological testing is highly specific.

The high degree of cross-reactivity of currently available serological flavivirus assays is a major issue of concern $[8,9]$. In Europe, TBEV is the most relevant flavivirus and TBE vaccination coverage ranges from $20 \%$ (southern Germany) to more than $80 \%$ (Austria) [10]. Of note, yellow fever vaccination is recommended for travellers to Brazil and other South American countries. In recent years, an estimated 300,000 to 350,000 travellers from Germany, Austria and Switzerland have visited Brazil and thus are currently at risk of having acquired ZIKV infection. Our results provide strong evidence that the Euroimmun ZIKV IgG ELISA is a specific tool and can be safely used to rule out ZIKV infection even on the background of pre-existing antibodies to different flaviviruses and other acute infections. Importantly, this also applies to dengue-positive sera as shown on a limited number of dengue virus antibody-containing sera from European travellers. However, more data is needed from regions where dengue is endemic, e.g. South America. Of note, IgM and IgG antibodies against ZIKV were unambiguously identified in positive patient sera. This ZIKV ELISA allows easy, specific and high-throughput testing of suspected cases. However, neutralising antibody detection assays remain the gold standard for diagnosis and evaluation of tests, although they are restricted to specialist laboratories and allow low to medium throughput only [11]. Clearly, further studies are needed to determine the sensitivity of the assay using a larger set of samples taken at different time points of the infection. Alternatively, a limited number of other commercial ZIKV serology tests are on the market or will be available in short time, but extensive validation data is pending to date.

The ZIKV ELISA may primarily aid gynaecologists and clinicians in travel medicine in the diagnosis of recent ZIKV infection and public health officials in developing guidelines on diagnostic algorithms for ZIKV infection. Interestingly, in the acute phase, testing of saliva samples using RT-PCR can increase the detection rate as seen in our German tourist and as reported elsewhere [12]. Of note, acute EBV can cause false positive IgM reactions in the ZIKV IgM ELISA, owing to a polyclonal stimulation of $B$ cells, which makes it necessary to rule out acute EBV infection in ambiguous cases [13]. This was also seen in our results.

All sera with high antibody titres were retrieved from our local biobank. The availability of well-defined sera to validate novel assays is important for emerging pathogens [14]. Thus, collecting and sharing of sera by (national) laboratories should be promoted to strengthen preparedness for emerging diseases. 


\section{Conclusion}

We provide evidence that the Euroimmun ELISA is highly specific and reliable when used for patients with previous flavivirus exposure or vaccination. This also applies to TBEV, which is of particular relevance for European patients. This diagnostic tool will aid in counselling patients, pregnant women and travellers after returning from ZIKV-endemic regions to Europe.

\section{Conflict of interest}

None declared.

\section{Authors' contributions}

$\mathrm{DH}$, JSC, and MP wrote the manuscript. IH performed the laboratory investigation, JSC provided ZIKV patient sera. All authors participated in the investigation. All authors read and approved the final manuscript.

\section{References}

1. European Centre for Disease Prevention and Control (ECDC) Zika virus infection: Factsheet for health professionals. Stockholm: ECDC. [Accessed: 28 Mar 2016]. Available from: http://ecdc.europa.eu/en/healthtopics/zika_virus_infection/ factsheet-health-professionals/Pages/factsheet_health_ professionals.aspx\#sthash.Ovu33Mb6.dpuf

2. Vorou R. Letter to the editor: diagnostic challenges to be considered regarding Zika virus in the context of the presence of the vector Aedes albopictus in Europe. Euro Surveill. 2016;21(10):30161. http://dx.doi.org/DOI: 10.2807/1560-7917. ES.2016.21.10.30161

3. Serological diagnosis of Zika virus infections. Press release. Luebeck: Euroimmun; 2016. Available from: https://www. euroimmun.com/index.php?elD=dumpFile \& $\mathrm{t}=\mathrm{f} \& \mathrm{f}=3013$ \&token =6ccobb2eb7c10f484ae50ad91e69cd6424088529

4. Tappe D, Rissland J, Gabriel M, Emmerich P, Gunther S, Held G, et al. First case of laboratory-confirmed Zika virus infection imported into Europe, November 2013. Euro Surveill. 2014;19(4):20685. DOI: 10.2807/1560-7917.ES2014.19.4.20685 PMID: 24507467

5. Brasil P, Pereira JP, Raja Gabaglia C, Damasceno L, Wakimoto M, Ribeiro Nogueira RM, et al. Zika Virus Infection in Pregnant Women in Rio de Janeiro - Preliminary Report. N Engl J Med. 2016;NEJMoa1602412. DOI: 10.1056/NEJMoa1602412 PMID: 26943629

6. Rasmussen SA, Jamieson DJ, Honein MA, Petersen LR. Zika Virus and Birth Defects - Reviewing the Evidence for Causality.N Engl J Med. 2016;NEJMsr1604338. DOI: 10.1056/ NEJMsr1604338 PMID: 27074377

7. Zika virus infection - short facts assembled by the Society for Virology in Germany. Erlangen: European Society for Virology [Accessed: 19 Apr 2016]. Available from: http://www.eusv.eu/ zika-virus-infection-short-facts-assembled-by-the-society-forvirology-in-germany/

8. Allwinn R, Doerr HW, Emmerich P, Schmitz H, Preiser W. Crossreactivity in flavivirus serology: new implications of an old finding?Med Microbiol Immunol (Berl). 2002;190(4):199-202. DOI: 10.1007/s00430-001-0107-9 PMID: 12005333

9. Koraka P, Zeller H, Niedrig M, Osterhaus AD, Groen J. Reactivity of serum samples from patients with a flavivirus infection measured by immunofluorescence assay and ELISA.Microbes Infect. 2002;4(12):1209-15. DOI: 10.1016/S1286-4579(02)016477 PMID: 12467761

10. Heinz FX, Stiasny K, Holzmann H, Grgic-Vitek M, Kriz B, Essl $A$, et al. Vaccination and tick-borne encephalitis, central Europe. Emerg Infect Dis. 2013;19(1):69-76. DOI: 10.3201/ eid1901.120458 PMID: 23259984

11. Venturi G, Zammarchi L, Fortuna C, Remoli ME, Benedetti E, Fiorentini C, et al. Authors' reply: diagnostic challenges to be considered regarding Zika virus in the context of the presence of the vector Aedes albopictus in Europe. Euro Surveill. 2016;21(10):30163. DOI: 10.2807/1560-7917. ES.2016.21.10.30163 PMID: 26988337
12. Musso D, Roche C, Nhan TX, Robin E, Teissier A, Cao-Lormeau VM. Detection of Zika virus in saliva.J Clin Virol. 2015;68:53-5. DOI: 10.1016/j.jcv.2015.04.021 PMID: 26071336

13. Alidjinou EK, Lazrek M, Libier L, Bocket L, Dewilde A, Engelmann I, et al. A patient with fever, abdominal pain and bicytopenia: Trouble once again with these IgM antibodies! J Clin Virol. 2016;75:60-3. DOI: 10.1016/j.jcv.2015.11.025 PMID: 26679224

14. Reusken C, Mou H, Godeke GJ, van der Hoek L, Meyer $B$, Müller MA, et al. Specific serology for emerging human coronaviruses by protein microarray. Euro Surveill. 2013;18(14):20441. DOI: 10.2807/1560-7917. ES2013.18.14.20441 PMID: 23594517

\section{License and copyright}

This is an open-access article distributed under the terms of the Creative Commons Attribution (CC BY 4.0) Licence. You may share and adapt the material, but must give appropriate credit to the source, provide a link to the licence, and indicate if changes were made.

This article is copyright of the authors, 2016. 